\title{
Chromatin-Associated Protein Complexes Link DNA Base J and Transcription Termination in Leishmania
} \author{
Marilyn Parsons, ${ }^{a, e, f}$ (D) Peter J. Mylera,b,c,e,f \\ aCenter for Global Infectious Disease Research, Seattle Children's Research Institute, Seattle, Washington, USA \\ bSeattle Structural Genomics Center for Infectious Disease, Seattle, Washington, USA \\ 'Department of Biomedical Informatics and Medical Education, University of Washington, Seattle, Washington, USA \\ Institute for Systems Biology, Seattle, Washington, USA \\ eDepartment of Pediatrics, University of Washington, Seattle, Washington, USA \\ fDepartment of Global Health, University of Washington, Seattle, Washington, USA
}

Bryan C. Jensen, ${ }^{a}$ Isabelle Q. Phan, ${ }^{a, b}$ Jacquelyn R. McDonald, ${ }^{a}$ Aakash Sur, ${ }^{a, c}$ Mark A. Gillespie, ${ }^{d *}$ Jeffrey A. Ranish, ${ }^{d}$

ABSTRACT Unlike most other eukaryotes, Leishmania and other trypanosomatid protozoa have largely eschewed transcriptional control of gene expression, relying instead on posttranscriptional regulation of mRNAs derived from polycistronic transcription units (PTUs). In these parasites, a novel modified nucleotide base ( $\beta$-D-glucopyranosyloxymethyluracil) known as J plays a critical role in ensuring that transcription termination occurs only at the end of each PTU, rather than at the polyadenylation sites of individual genes. To further understand the biology of J-associated processes, we used tandem affinity purification (TAP) tagging and mass spectrometry to reveal proteins that interact with the glucosyltransferase performing the final step in J synthesis. These studies identified four proteins reminiscent of subunits in the PTW/PP1 complex that controls transcription termination in higher eukaryotes. Moreover, bioinformatic analyses identified the DNA-binding subunit of Leishmania PTW/PP1 as a novel J-binding protein (JBP3), which is also part of another complex containing proteins with domains suggestive of a role in chromatin modification/remodeling. Additionally, JBP3 associates (albeit transiently and/or indirectly) with the trypanosomatid equivalent of the PAF1 complex involved in the regulation of transcription in other eukaryotes. The downregulation of JBP3 expression levels in Leishmania resulted in a substantial increase in transcriptional readthrough at the $3^{\prime}$ end of most PTUs. We propose that JBP3 recruits one or more of these complexes to the J-containing regions at the end of PTUs, where they halt the progression of the RNA polymerase. This decoupling of transcription termination from the splicing of individual genes enables the parasites' unique reliance on polycistronic transcription and posttranscriptional regulation of gene expression.

IMPORTANCE Leishmania parasites cause a variety of serious human diseases, with no effective vaccine and emerging resistance to current drug therapy. We have previously shown that a novel DNA base called $J$ is critical for transcription termination at the ends of the polycistronic gene clusters that are a hallmark of Leishmania and related trypanosomatids. Here, we describe a new J-binding protein (JBP3) associated with three different protein complexes that are reminiscent of those involved in the control of transcription in other eukaryotes. However, the parasite complexes have been reprogrammed to regulate transcription and gene expression in trypanosomatids differently than in the mammalian hosts, providing new opportunities to develop novel chemotherapeutic treatments against these important pathogens.
Citation Jensen BC, Phan IQ, McDonald JR, Sur A, Gillespie MA, Ranish JA, Parsons M, Myler PJ. 2021. Chromatin-associated protein complexes link DNA base $J$ and transcription termination in Leishmania. mSphere 6:e01204-20. https:// doi.org/10.1128/mSphere.01204-20.

Editor William J. Sullivan, Jr., Indiana University School of Medicine

Copyright $\odot 2021$ Jensen et al. This is an open-access article distributed under the terms of the Creative Commons Attribution 4.0 International license.

Address correspondence to Peter J. Myler peter.myler@seattlechildrens.org. * Present address: Mark A. Gillespie, Allen Institute, Seattle, Washington, USA.

Received 25 November 2020

Accepted 27 January 2021

Published 24 February 2021 
- he genus Leishmania includes several species of protozoan parasites that cause a spectrum of human diseases, ranging from cutaneous lesions to disfiguring mucocutaneous and lethal visceral leishmaniasis, depending primarily on the species involved. Leishmania is transmitted through the bite of the sand fly and belongs to the family Trypanosomatidae, which also includes the vector-borne human pathogens Trypanosoma brucei, the causative agent of human African trypanosomiasis (African sleeping sickness), and Trypanosoma cruzi, the causative agent of Chagas' disease. Reflecting the ancient divergence of these organisms, the Trypanosomatidae exhibit a myriad of biological differences from "higher" eukaryotes. One major difference is that each chromosome is organized into a small number of polycistronic transcription units (PTUs), which consist of tens to hundreds of protein-coding genes cotranscribed from a single initiation site at the $5^{\prime}$ end of the PTU to a termination site at the $3^{\prime}$ end (1). Interestingly, unlike the operons of prokaryotes, genes within each PTU are not confined to a single pathway or function. Individual genes within the primary transcript are trans-spliced by the addition of a 39-nucleotide spliced-leader (SL) miniexon to provide the $5^{\prime}$ cap-4 structure and polyadenylated to form the mature individual mRNAs. As a result of this unique genomic organization, all genes within a PTU are transcribed at the same rate $(2,3)$. Hence, gene expression must be controlled by posttranscriptional processes such as the splicing/polyadenylation rate, RNA stability, and translational regulation.

A second distinct feature of the Trypanosomatidae (and other Euglenozoa) is that $\sim 1 \%$ of the thymidine bases in the nuclear genome are glucosylated to form the novel nucleotide $\beta$-D-glucopyranosyloxymethyluracil, usually referred to as $J(4,5)$. While the majority of $J$ is localized within telomeric repeat sequences $(4,6-9)$, chromosome-internal $J$ is found at almost all transcription termination sites (TTSs) $(10,11)$ and centromeres, which also correspond to the major replication origins on Leishmania chromosomes $(12,13)$. In some trypanosomatids (although not Leishmania), J is also found in transcriptionally silent regions containing retrotransposons and/or other repetitive sequences (9). J biosynthesis occurs in two steps, whereby one of two proteins (JBP1 or JBP2) hydroxylates the methyl group of thymidine to form hydroxymethyldeoxyuracil (HmdU), which is subsequently further modified by a glucosyltransferase (HmdUGT) to form J $(10,14)$. Both JBP1 and JBP2 have an N-terminal oxygenase (Tet_JBP) catalytic domain, but JBP1 contains a central J-binding domain (15), while JBP2 instead contains SNF2_N, ATP-binding, and helicase C-terminal domains that are suggestive of a role in chromatin binding and/or remodeling $(16,17)$. Null mutants of JBP1 have not been isolated (despite multiple attempts) in Leishmania, suggesting that it is an essential gene (and, hence, that $\mathrm{J}$ is required for viability) (18). In contrast, jbp2-null mutants have been isolated and shown to have 40\% less J than wild-type (WT) parasites (11). Chromosome-internal J was gradually lost during continuous growth of Leishmania tarentolae jbp2-null mutants, with a concomitant increase in readthrough transcription at termination sites, suggesting a critical role for J (and JBP2) in transcription termination. Coupled with the identification of a JBP1 recognition motif (19), these data led to the model that JBP1 is responsible for J maintenance after DNA replication by binding to preexisting $J$ on the parental strand and modifying a thymidine 12 nucleotides downstream on the newly synthesized strand. Conversely, JBP2 is proposed to be largely responsible for de novo synthesis of $J$ when JBP1 is not able to fully restore $J$ on both strands.

Despite this knowledge of the enzymes involved in J biosynthesis, we currently know little about how J mediates transcription termination (and/or repression of initiation) since the machinery underlying this process has not yet been identified. Here, we have used tandem affinity purification (TAP) tagging and tandem mass spectrometry (MS/MS) to identify a PTW/PP1-like protein complex that interacts with HmdUGT. This complex includes a novel J-binding protein (JBP3) that appears to be essential in Leishmania. Transcriptome sequencing (RNA-seq) analysis following conditional downregulation of JBP3 expression shows substantially higher levels of transcriptional 


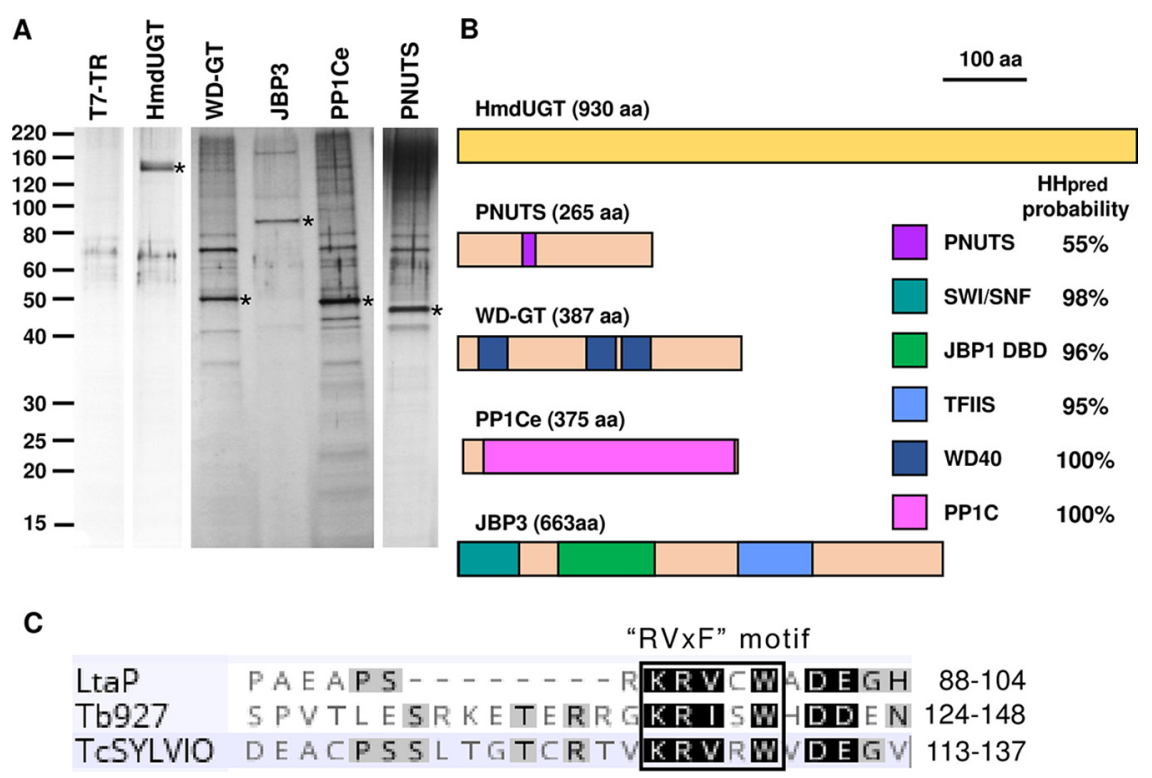

FIG 1 The PJW/PP1 complex. (A) Proteins in the peak fractions from TAPs of HmdUGT and other components of the PJW/PP1 complex (WD-GT, JBP3, PP1Ce, and PNUTS) overexpressed in T7-TR cells were separated by 4 to 20\% SDS-PAGE and silver stained. Each lane represents $5 \%$ of the total fraction. The TAP-tagged "bait" protein is indicated by an asterisk. The first lane shows the equivalent fraction from a mock purification of control (T7-TR) cells. (B) Schematic representation of key domains in the four proteins (and GT) in the PJW/PP1 complex, as predicted by HHpred analysis. The probability of a match between the Leishmania protein and the most similar experimentally determined structure is shown to the right. Potential functions of each domain are discussed in the text. (C) The putative PP1C-interacting domain of L. donovani (LtaP), T. brucei (Tb927), and T. cruzi (TCSYLVIO) PNUTS is shown, and the "RVXF" docking motif (which has a consensus sequence of K/R-K/R-V/I-X-F/W, where $X$ is a residue other than $F, I$, M, Y, D, or P) (21) is shown at the top. The amino acid (aa) positions for each end of the domain are shown to the right.

readthrough at the $3^{\prime}$ end of most PTUs, suggesting that it plays an important role in transcription termination. While the manuscript was being prepared, Kieft et al. (20) reported similar results in Leishmania and T. brucei. Here, we have extended these findings by demonstrating that JBP3 also interacts with another protein complex likely involved in chromatin modification/remodeling as well as, to a lesser degree, with an RNA polymerase II (RNAP II)-associated factor 1 complex (PAF1C)-like complex that likely interacts with RNAP II. Therefore, despite the differences in gene regulation from other eukaryotes, Leishmania appears to utilize proteins related to those used for chromatin remodeling and transcriptional regulation in other eukaryotes to provide the molecular machinery that links $\mathrm{J}$ to the termination of RNAP II-mediated transcription.

\section{RESULTS}

Identification of a protein complex containing a novel J-binding protein. To date, only three proteins have been shown to be involved in J biosynthesis: JBP1, JBP2, and HmdUGT (referred to here as GT). To expand the network of proteins important in $\mathrm{J}$ biosynthesis and/or function, we used mass spectrometry to identify proteins that copurified with a TAP-tagged GT bait expressed in L. tarentolae. Two separate experiments were performed: the first used extracts from wild-type parasites constitutively expressing the tagged protein, while the second pulldown (and all other TAP-tag experiments) were performed using extracts from a tetracycline (Tet)-induced T7-TR cell line, which overexpresses the tagged protein integrated at the ODC locus (see Fig. S1A in the supplemental material). After affinity purification, SDS-PAGE and silver staining confirmed the successful enrichment of the bait protein in the pooled eluates (Fig. 1A). Proteins were identified by liquid chromatography-tandem mass spectrometry (LC-MS/MS), and their ( $\log _{2}$ fold) enrichment was calculated by comparison to a 
TABLE 1 Enrichment ${ }^{a}$ of proteins in the PJW/PP1 complex

\begin{tabular}{|c|c|c|c|c|c|c|c|}
\hline \multirow[b]{2}{*}{ Gene ID } & \multirow[b]{2}{*}{ Gene name } & \multirow[b]{2}{*}{ HHpred/InterPro domain(s) } & \multicolumn{5}{|c|}{ TAP-tagged protein } \\
\hline & & & HmdUGT & PP1Ce & PNUTS & JBP3 & WD-GT \\
\hline LtaP36.2450 & HmdUGT & None found & 14.1 & 7.2 & 8.2 & 10.6 & 9.2 \\
\hline LtaP33.1440 & PNUTS & PPP1R10/PNUTS & (9.8) & 12.5 & 17.7 & 10.3 & 13.2 \\
\hline LtaP36.0380 & JBP3 & SWI1, J-binding, and N-terminal TFIIS & 11.1 & 12.3 & 10.3 & 15.9 & 12.5 \\
\hline LtaP32.3990 & WD-GT & WD40 repeats & 9.3 & 14.8 & 12.7 & 12.3 & 16.3 \\
\hline
\end{tabular}

${ }^{a}$ Enrichment is expressed as the mean $\log _{2}$ fold change compared to control pulldowns. Parentheses indicate that enrichment was observed in only one replicate.

control (untransfected) parental cell line (see Data Set S1 in the supplemental material for a complete list of all proteins detected in each TAP tag pulldown).

The data revealed substantial ( $>500$-fold) enrichment of five proteins (including the "bait") in the GT-TAP pulldown: four that were enriched in both replicates and one that was detected only in the first experiment (Table 1). These results suggested that the five proteins form a GT-associated protein complex, which was investigated in more detail, as described below. In addition, six prefoldin subunits were enriched by 50 - to 450-fold in one or both replicates (see Table S1 in the supplemental material, which includes all proteins that were substantially enriched in each pulldown). However, since prefoldin likely acts only as a chaperone for one or more proteins of the GT-associated complex, these proteins are not further considered here.

One of the highly enriched proteins in the GT-TAP pulldown (LtaP15.0230) was annotated in TriTrypDB as a putative protein phosphatase 1 catalytic subunit (PP1C), while the other three (LtaP36.0380, LtaP33.1440, and LtaP32.3990) were all annotated as "hypothetical protein, conserved." Constructs were made for the expression of TAPtagged proteins and transfected into the L. tarentolae T7-TR strain. Affinity purification was performed on two (independently generated) cell lines for each version of the TAP-tagged proteins (the results from one replicate of each are shown in Fig. S1B) and analyzed by LC-MS/MS (Data Set 1). The results from these pulldowns (Table 1 and Table S1) show that the four identified proteins described above were all highly enriched (as was GT, albeit to a lesser degree). Thus, we conclude that these proteins form a stable complex, with GT perhaps being more transiently associated than the other four components.

LtaP15.0230 encodes one of eight isoforms of PP1C found in the L. tarentolae genome. Phylogenetic analysis (Fig. S2) indicates that there are five different clades of PP1C paralogues in trypanosomatids, and PP1Ce (encoded by LtaP15.0230) belongs to a clade that lacks any mammalian orthologue. Interestingly, salivarian trypanosomes (including $T$. brucei) also lack PP1Ce, although it is present in stercorarian trypanosomes (Fig. S2) and the more distantly related kinetoplastids Blechomonas ayalai, Paratrypanosoma confusum, and Bodo saltans (data not shown). TAP tagging of PP1Ce resulted in the copurification of seven proteins with $>150$-fold enrichment (Table S1). These include GT and the other three components of the complex described above as well as three proteins (encoded by LtaP05.1290, LtaP07.0770, and LtaP29.0170) annotated as protein phosphatase regulatory subunits (PPP1R7/Sds22, PPP1R11/inhibitor 3, and PPP1R2/inhibitor 2, respectively). In higher eukaryotes, PPP1R2 and PPP1R11 use the same "RVxF" docking motif to bind to and inhibit PP1C, while PPPR7 docks at a different site (21). At least some mammalian PP1C proteins form an inactive heterotrimeric complex containing PP1R7 and PPP1R11 (22). Thus, we suggest that PP1Ce forms at least three separate complexes: one with both PPP1R7 and PPP1R11, a second with PPP1R2 (which may or may not contain PPP1R7), and the third being the GT-associated complex, as described below.

We performed a series of bioinformatic analyses to identify domains and/or motifs that might provide hints as to the function of the three proteins of the GT-associated complex that lack a functional description. BLASTP and InterProScan searches showed high-confidence matches only to orthologues in other trypanosomatids with no 
informative domains identified. However, HHpred analysis, which detects remote protein homology by using hidden Markov models and structure prediction (23), revealed a number of structural matches, which are summarized in Fig. 1B and shown in detail in Fig. S3. HHpred predicts that the central portion (residues 88 to 105) of LtaP33.1440 contains structural similarity to a portion of human serine/threonine-protein phosphatase 1 regulatory subunit 10 (PPP1R10), also known as PNUTS (for PP1 nuclear targeting subunit) (24-27). The trypanosomatid protein is much smaller (264 versus 940 amino acids) than mammalian PNUTS, with the sequence and structural similarities restricted to the central region described above (Fig. S3A). However, this region contains the RVxF sequence motif (Fig. 1C) noted above, which is present in most inhibitors and responsible for interaction with PP1C (28). Therefore, in deference to precedent in the field (20), we refer to this protein as PNUTS despite the lack of an obvious nuclear localization signal. TAP-tagging of PNUTS showed significant enrichment of the four other components of the GT-associated protein complex and no other proteins (Table S1).

BLASTP searches of LtaP32.3990 returned matches to orthologues in other trypanosomatids as well as WD40 repeats in proteins from several other organisms, while HHpred analysis (Fig. S3B) identified at least three WD40 repeats. Therefore, we refer to this protein as WD-GT, to distinguish it from the numerous other WD40 repeat-containing proteins in Leishmania. TAP-tagged WD-GT pulled down the four other components of the GT-associated protein complex as well as a number of chaperone-associated proteins, including prefoldin, T-complex, and heat shock proteins (Table S1).

HHpred analysis of LtaP36.0380 revealed three separate domains with structural similarity to different proteins (Fig. S3C). The N-terminal domain (residues 2 to 86) is similar to the central portion of the SWI1 subunit of the Saccharomyces cerevisiae SWI/ SNF chromatin remodeling complex, while the C-terminal domain (residues 384 to 485 ) is related to the N-terminal TFIIS domain of mammalian PNUTS. Most importantly, the central portion (residues 137 to 269) is predicted to have substantial structural similarity to the DNA-binding domain (DBD) of JBP1, and in silico folding of this region revealed conservation of the functional signature $D-(W / F / Y)-x-x-G G T R Y$ motif present in all trypanosomatid JBP1 proteins (Fig. 2A). In addition, a structural model of the DBD from LtaP36.0380 contains a binding pocket large enough to accommodate the glucose ring of $\mathrm{J}$ (Fig. $2 \mathrm{~B}$ and $\mathrm{C}$ ), and preliminary experiments indicate that it binds preferentially to J (Anastasis Perrakis, NKI, Amsterdam, Netherlands, personal communication). While the manuscript was in preparation, this J-binding function of LtaP36.0380 was experimentally confirmed by others (20), so the protein was renamed $\underline{J}$-binding protein 3 (JBP3).

The molecular characteristics of the four proteins identified in the GT pulldowns, a PP1 catalytic subunit (PP1Ce), a predicted PP1 regulatory protein (PNUTS), a WD40 repeat protein (WD-GT), and a DNA-binding protein (JBP3), are highly reminiscent of the components of the mammalian PTW/PP1 complex. This complex, which contains PP1C, PNUTS, WDR82, and the DNA-binding protein TOX4, has a role in controlling chromatin structure $(27,29)$. Importantly, the mammalian PTW/PP1 complex was recently found to negatively regulate the RNAP II elongation rate by dephosphorylating the transcription elongation factor Spt5, leading to transcription termination at polyadenylation sites (30). Thus, our results indicate that GT associates with a PTW/ PP1-like complex in Leishmania (which we refer to as PJW/PP1), wherein JBP3 replaces the DNA-binding function of TOX4.

JBP3 is part of another chromatin remodeling complex. While tandem affinity purification of TAP-tagged JBP3 showed $>256$-fold enrichment of the PJW/PP1 complex proteins (PP1Ce, PNUTS, WD-GT, and GT) (Table S1), another four proteins (encoded by LtaP35.2400, LtaP28.2640, LtaP12.0900, and LtaP14.0150) were >3,000-fold enriched (Table 2). BLASTP analyses of these proteins failed to reveal convincing matches to anything other than orthologues in other trypanosomatids, and InterProScan showed no matches using the default parameters. However, LtaP35.2400 is annotated as a "SET domain-containing protein, putative," in TriTrypDB, and HHpred 
A

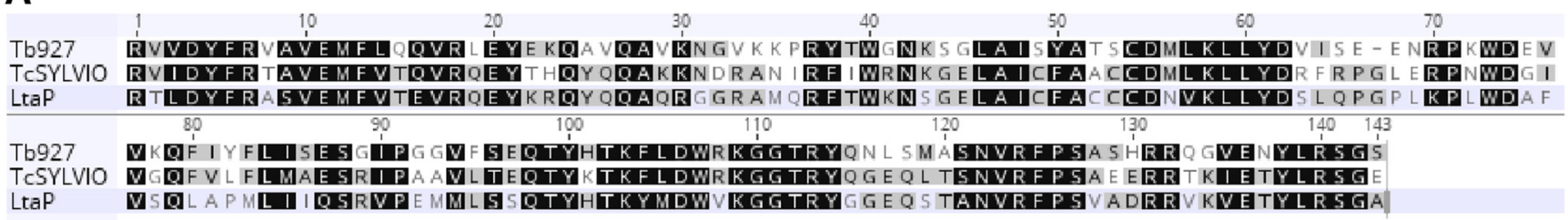

B

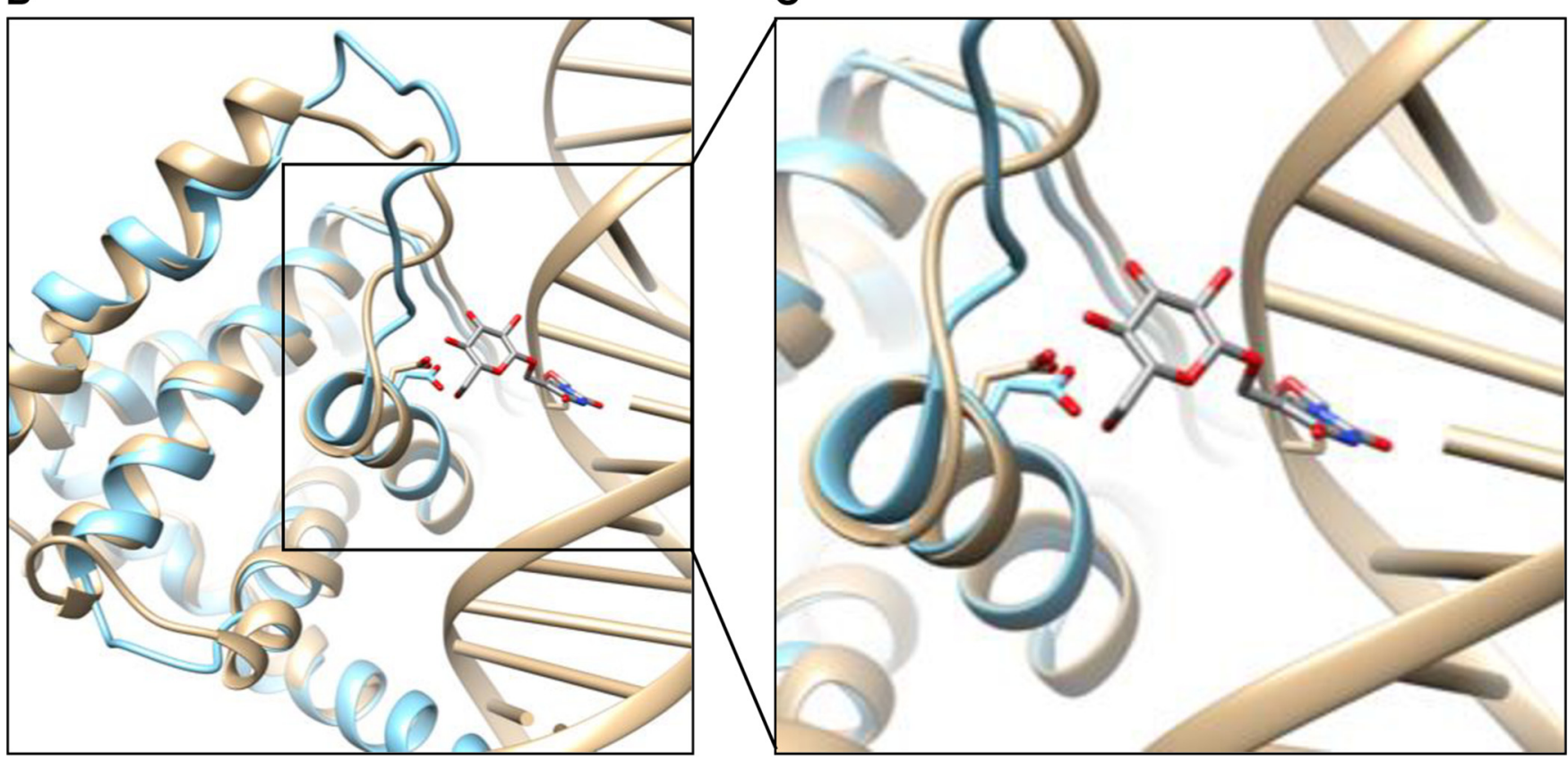

FIG 2 Modeling of the JBP3 DNA-binding domain. (A) Sequence alignment of the putative JBP3 J-binding domains from the T. brucei EATRO927 strain, the $T$. cruzi Silvio strain, and the L. tarentolae Parrot strain. Residues that are identical or conservatively replaced in all three species are shaded black, while those that are identical or conserved in two species are shaded gray. (B) The structure of the DNA-binding domain from JBP3 (light blue) was modeled using RosettaCM against the J-binding domain of JBP1 (tan) from PDB accession number 2XSE. The interaction between the conserved aspartic acid residue $\left(\mathrm{Asp}_{525}\right.$ in $\mathrm{JBP} 1$ and $\mathrm{Asp}_{241}$ in JBP3) and the glucose of base $\mathrm{J}$ is shown. (C) Higher-resolution view of the interaction between the conserved aspartate of both proteins and base $J$.

analysis revealed that the $\mathrm{N}$-terminal region (amino acids 70 to 227) contains structural similarity to SET domain-containing proteins, while the central portion (residues 355 to 385) shows weaker similarity to C4-type zinc finger domains from several unrelated proteins (Fig. S3D). SET domains, which are usually involved in binding to and/or methylation of histones (31), are also present in several other Leishmania proteins, so we have named this protein SET-J3C to distinguish it from the others. HHpred analysis of LtaP14.0150 showed structural similarity to chromatin organization modifier (Chromo) domains $(32,33)$ from numerous eukaryotic proteins at its $\mathrm{N}$ terminus (amino acids 1 to 55) and weak similarity to Chromo shadow domains at the $C$ terminus (34) (Fig. S3E). Therefore, we dubbed this protein Chromo-J3C and predict that it may be involved in

TABLE 2 Enrichment ${ }^{a}$ of proteins in the JBP3-associated chromatin remodeling complex

\begin{tabular}{|c|c|c|c|c|c|}
\hline \multirow[b]{2}{*}{ Gene ID } & \multirow[b]{2}{*}{ Gene name } & \multirow[b]{2}{*}{ HHpred/InterPro domain(s) } & \multicolumn{3}{|c|}{ TAP-tagged protein } \\
\hline & & & JBP3 & J3C-Chromo & J3C-CS \\
\hline LtaP36.0380 & JBP3 & SWI1, J-binding, and N-terminal TFIIS & 15.9 & 7.9 & 7.8 \\
\hline LtaP35.2400 & SET-J3C & SET and C4-type Zn finger & 12.8 & 8.2 & 9.4 \\
\hline LtaP14.0150 & Chromo-J3C & Chromo and Chromo shadow & 11.7 & 11.6 & 8.8 \\
\hline LtaP28.2640 & CS-J3C & Chromo shadow & 12.5 & 9.8 & 11.8 \\
\hline LtaP12.0900 & HPC-J3C & None found & 12.1 & 9.8 & 6.3 \\
\hline
\end{tabular}

${ }^{a}$ Enrichment is expressed as the mean $\log _{2}$ fold change compared to control pulldowns. 


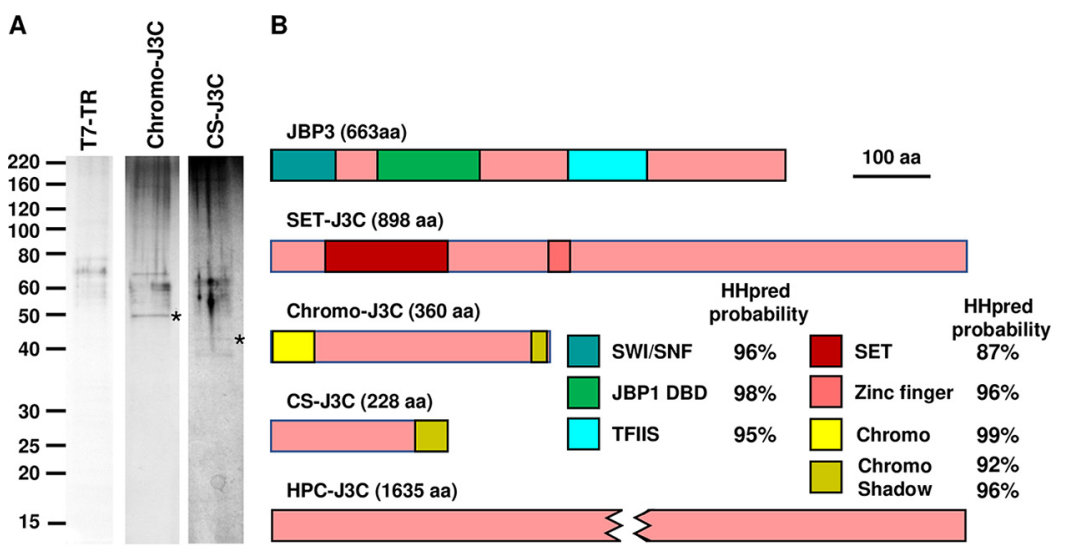

FIG 3 The JBP3-associated chromatin complex. (A) Proteins that copurify with TAP-tagged ChromoJ3C and CS-J3C overexpressed in T7-TR cells were analyzed by SDS-PAGE and silver staining as described in the legend of Fig. 1. (B) Schematic representation showing the key domains of the five proteins that copurified in the JBP3-associated chromatin (J3C) complex.

the recognition of methylated lysine residues on histone tails. LtaP28.2640 is annotated as a "hypothetical protein, conserved," but residues 187 to 227 also show structural similarity to the Chromo shadow domain (Fig. S3F), and so we called it CS-J3C. The LtaP12.0900 gene is misassembled in the L. tarentolae reference genome, so we used full-length orthologues from other Leishmania genomes for subsequent analyses. However, BLASTP, InterProScan, and HHpred analyses were uninformative, so we called this protein HPC-J3C (for hypothetical protein, conserved in J3C). Phylogenetic analysis showed that HPC-J3C has poor sequence conservation, even in other trypanosomatids, with orthologues in other genera being shorter than in Leishmania. HHpred analysis of the $T$. brucei orthologue (Tb927.1.4250) showed structural similarity at the $C$ terminus to subunits from several large protein complexes involved in a variety of processes, including histone remodeling (Fig. S3G).

To confirm the association of these proteins with JBP3 (and each other), we transfected TAP-tagged versions of them into $L$. tarentolae T7-TR. Unfortunately, cloning of $H P C-J 3 C$ failed because of errors in the genome sequence (see above), and transfectants containing the SET-J3C construct did not express the tagged protein (perhaps because overexpression was deleterious for cell growth), but the Chromo-J3C and CS-J3C transfectants expressed tagged proteins of the expected size (Fig. S1B), enabling affinity purification (Fig. 3A). Subsequent mass spectrometric analysis of copurifying proteins showed that Chromo-J3C and CS-J3C pulldowns enriched JBP3 and the same JBP3-associated proteins (Table 2 and Table S1), indicating that they form a separate JBP3-containing complex. Bioinformatic analyses (described above) suggest that the proteins in this complex are likely associated with chromatin modification and/or remodeling, prompting us to call it the JBP3-associated chromatin complex (abbreviated J3C).

JBP3 interacts with PAF1C. In addition to the components of the PJW/PP1 and J3C complexes, two other proteins (encoded by LtaP35.2870 and LtaP29.1270) were substantially enriched ( $>110$-fold and $>23$-fold, respectively) in both JBP3 TAP tag experiments (Table 3 and Table S1). LtaP35.2870 is annotated in TriTrypDB as "RNA polymerase-associated protein LEO1, putative," and this homology was confirmed by HHpred analyses (Fig. S3H). LEO1 is a subunit of the RNAP II-associated factor 1 complex (PAF1C), which facilitates transcription elongation by regulating chromatin modification (35-37). Interestingly, mass spectrometric analysis of proteins that copurified with TAP-tagged LEO1 (Fig. 4A and Table 3) did not detect JBP3 but identified three proteins (encoded by LtaP36.4090, LtaP29.2750, and LtaP29.1270) that were enriched $>630$-fold in both experiments (Table S1). The first two were also enriched $>50$-fold in one of the two JBP3 TAP tag experiments (Table S1) and are obvious homologues of 
TABLE 3 Enrichment ${ }^{a}$ of proteins in the PAF1C-L complex

\begin{tabular}{|c|c|c|c|c|}
\hline \multirow[b]{2}{*}{ Gene ID } & \multirow{2}{*}{$\begin{array}{l}\text { Gene } \\
\text { name }\end{array}$} & \multirow[b]{2}{*}{ HHpred/InterPro domain } & \multicolumn{2}{|c|}{$\begin{array}{l}\text { TAP-tagged } \\
\text { protein }\end{array}$} \\
\hline & & & JBP3 & LEO1 \\
\hline LtaP35.2870 & LEO1 & Leo1-like protein & 7.4 & 16.8 \\
\hline LtaP29.1270 & DCNL & PONY/DCUN1 domain & 5.4 & 13.9 \\
\hline LtaP36.4090 & CDC73 & Cell division control protein 73 (C terminal) & $(5.7)$ & 10.4 \\
\hline LtaP29.2750 & CTR9 & RNA polymerase-associated protein Ctr9 & $(6.2)$ & 9.5 \\
\hline LtaP14.0860 & RTF1L & Plus-3 domain & 0.6 & 3.8 \\
\hline
\end{tabular}

${ }^{a}$ Enrichment is expressed as the mean $\log _{2}$ fold change compared to control pulldowns. Parentheses indicate that enrichment was observed in only one replicate.

PAF1C subunits. HHpred analyses showed that LtaP36.4090 contains the Ras-like fold characteristic of the C-terminal domain of the CDC73 subunit of PAF1C (Fig. S3I) and is annotated as such in TriTrypDB. LtaP29.2750 contains several tetratricopeptide repeat (TPR) domains implicated in protein-protein interactions and shows considerable overall similarity to the CTR9 subunit of PAF1C (Fig. S3J). Functional studies of the T. brucei CTR9 orthologue (Tb927.3.3220) indicated that it is essential for parasite survival, and the depletion of its $m$ RNA reduced the expression of many genes involved in the regulation of mRNA levels (38). A fourth protein (LtaP29.1270) that was also substantially enriched in the TAP-tagged JBP3 experiments is not an obvious orthologue of any known PAF1C subunit. This protein is annotated as a "hypothetical protein, conserved," in TriTrypDB, but HHpred analysis (Fig. S3K) revealed a central domain (amino acids 232 to 358 ) with structural similarity to the PONY/DCUN1 domain found in DCN (defective in cullin neddylation) proteins that are involved in the regulation of ubiquitin ligation cascades (39). Our results (Table 3) suggest that LtaP29.1270, which we refer to as DCNL (DCN-like) here, forms an integral part (along with LEO1, CDC73, and CTRL) of a PAF1C-like (PAF1C-L) complex in Leishmania. The lack of significant reciprocal enrichment of JBP3 in the LEO1-TAP pulldowns (this study) and its absence from the CTR9TAP pulldowns in T. brucei (38) suggest a transient and/or indirect association of JBP3 with PAF1C-L.

TAP tagging of $T$. brucei CTR9 by others (38) revealed the same constellation of PAF1C-L subunits (LEO1, CDC73, and DCNL) as well as an additional protein (Tb927.7.4030). Close examination of our results revealed that the Leishmania orthologue (LtaP14.0860) of Tb927.7.4030 is also enriched >10-fold in both LEO1 TAP tag experiments (Table 3 and Table S1). While this protein is annotated as a "hypothetical protein, conserved," in TriTrypDB, HHpred analysis revealed N-terminal (amino acids 2 to 152)

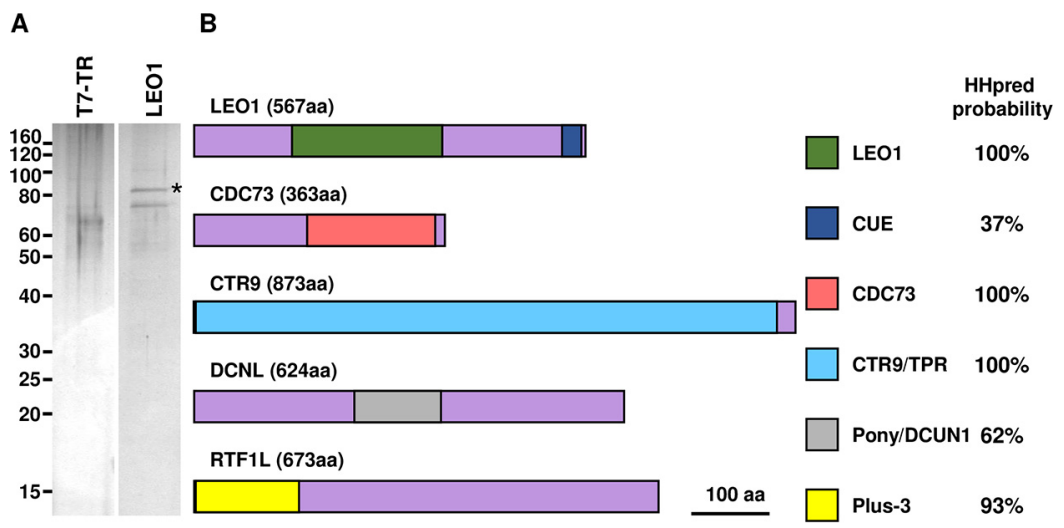

FIG 4 The PAF1C-like complex. (A) Proteins that copurified with TAP-tagged LEO1 overexpressed in T7-TR cells were analyzed by SDS-PAGE and silver staining as described in the legend of Fig. 1. (B) Schematic representation showing the key domains of five components of the PAF1C-L complex and their probability scores from HHpred analysis. 
structural similarity to the Plus-3 domain of human RTF1 (Fig. S3L), a component of human and yeast PAF1C. Thus, LtaP14.0860 (which we have dubbed RTF1L) is likely the functional equivalent of RTF1, although in Leishmania, it may be less tightly associated with PAF1C-L.

Depletion of JBP3 causes defects in transcription termination. The results of the TAP-tag experiments presented above suggest that JBP3 is an integral part of two protein complexes (PJW/PP1 and J3C) and interacts, possibly indirectly, with another (PAF1C-L). Since similar complexes have been associated with chromatin modification/ remodeling and regulation of transcription in other organisms, we postulated that JBP3 may mediate transcription termination in Leishmania. To test this hypothesis, we used CRISPR/Cas9 (40) to delete JBP3 in the L. tarentolae cell line bearing a tetracycline (Tet)-regulated copy of JBP3-TAP (see above). We were able to delete both endogenous copies of JBP3 only when JBP3-TAP expression was induced with Tet, suggesting that it is essential in Leishmania. To interrogate the effect of JBP3 depletion, we grew two stable transfectants lacking both endogenous copies of JBP3 (but containing a Tet-regulated copy of JBP3-TAP) for 8 to 11 days in the presence or absence of tetracycline (Fig. 5A). Cells grown in the presence of drug maintained a constant growth rate (with a generation time of $\sim 9 \mathrm{~h}$ ) over the length of the experiment, while the growth rate in the absence of drug decreased after day 3, with the generation time increasing to $>20 \mathrm{~h}$ on day 6 , before returning to almost the parental wild-type rate after day 10 (Fig. 5A). JBP3-TAP protein levels decreased markedly during the first day after the removal of tetracycline, dropping to $\sim 2 \%$ of the initial level by day 2 (Fig. $5 B$ ).

To assess the role of JBP3 in mRNA expression and transcription termination, RNA was isolated daily from the cells after Tet withdrawal and used to generate strand-specific RNA-seq libraries. Illumina sequencing reads were mapped to the $L$. tarentolae reference genome, and normalized read counts were calculated for every gene. Differential expression analysis revealed that JBP3-TAP mRNA levels were $\sim 20$-fold lower in the absence of Tet (Fig. 5C). Interestingly, there was a marked increase in the JBP3-TAP mRNA levels in the day 11 sample lacking Tet (Tet ${ }^{-}$sample), coincident with the resumption of normal growth. Consequently, this sample was excluded from subsequent analyses, along with the day $1 \mathrm{Tet}^{-}$samples (since JBP3 protein was still present at $\sim 8 \%$ of the initial level). Further analysis (using the DESeq 2 module of Geneious) revealed that 17 genes had significantly higher mRNA abundances ( $>2$-fold; $P<0.001)$ in the remaining Tet $^{-}$samples, which all had low JBP3 protein levels (Table S2). Interestingly, 14 of these genes are located adjacent (or close) to transcription termination sites (TTSs). Indeed, 34 of the 50 most upregulated genes are located near TTSs, with 24 of these located at convergent strand-switch regions (cSSRs) where the $3^{\prime}$ termini of two PTUs converge.

To further characterize these increases in RNA abundance, we analyzed the read coverage for $5 \mathrm{~kb}$ on either side of all 192 TTSs in the L. tarentolae genome. As

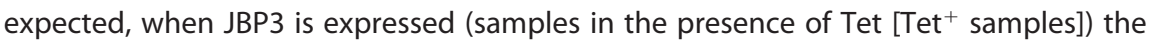
median-normalized coverage on the top (coding) strand decreased sharply downstream of the TTS (Fig. S4A). However, in samples with very low JBP3 protein levels (day 2 to $9 \mathrm{Tet}^{-}$), the read coverage downstream of the TTS was significantly higher, suggesting that reduction of JBP3 resulted in substantial transcriptional readthrough. Importantly, this increase in readthrough transcription did not occur to the same extent at different types of TTSs (Fig. 5D). It was most pronounced at the 23 noncentromeric cSSRs without RNA genes (Fig. 5E and Fig. S4B), where the transcript abundance was almost as high downstream of the TTS as it was upstream. There was also a significant increase in readthrough transcription downstream of the TTS between unidirectionally (head-to-tail) oriented PTUs (Fig. 5F and Fig. S4C), although it was more subtle since the gap between each PTU is small. Conversely, there was only a small increase in readthrough at TTSs upstream of RNA genes transcribed by RNAP III (Fig. S4D) and essentially no readthrough at centromeres (Fig. S4E) or telomeres (Fig. S4F). Analysis of bottom (noncoding) strand transcripts revealed no significant differences between 
A

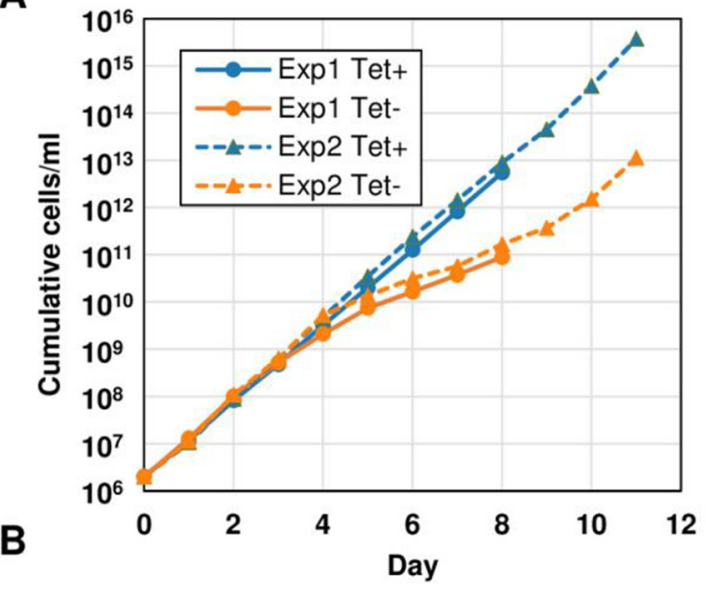

D
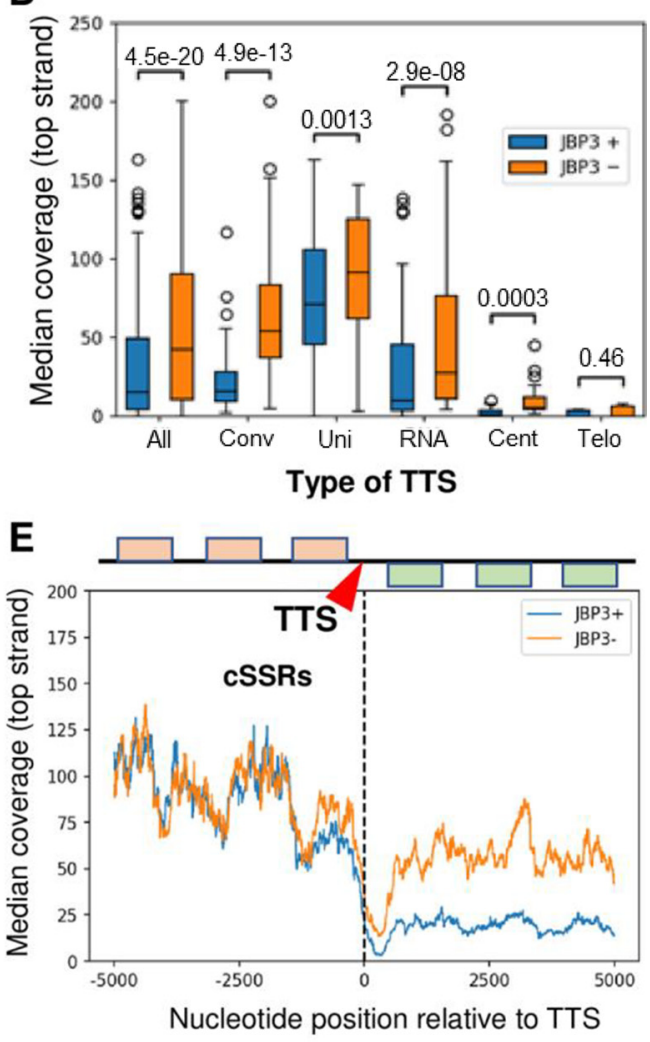

C

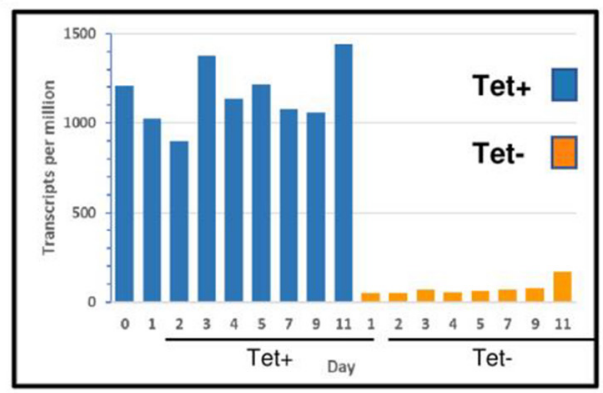

$\mathbf{F}$

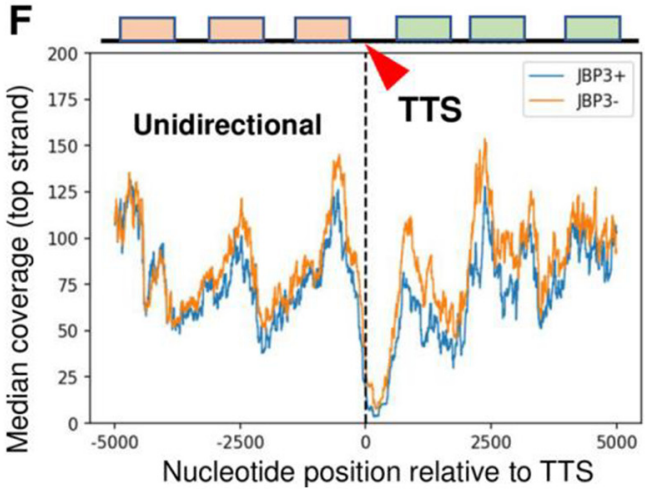

FIG 5 Depletion of JPB3 results in readthrough at transcription termination sites. (A) Growth analysis. Two independently generated $L$. tarentolae clones lacking endogenous JBP3 but containing a Tet-regulated TAP-tagged JBP3 gene were grown in the presence or absence of Tet. The numbers on the $y$ axis are corrected for dilution during subculturing. The solid lines show a clone where the JBP3 genes were replaced by pac (and grown in the presence of puromycin), while the dotted lines show a clone where the JBP3 genes were replaced by neo (and grown in G418). Exp1 and Exp2 refer to experiments 1 and 2, respectively. (B) The level of TAP-tagged JBP3 expressed by the T7-TR/JBP3MHTAP/ $\Delta j b p 3:: n e o$ clone grown in the absence of Tet was monitored by Western blotting using antibodies against the calmodulin-binding peptide (CBP) of the TAP tag. Antibodies against phosphoglycerate kinase (PGK) served as a loading control. The percent JBP3 levels in comparison to day 0 are shown below the anti-CBD blot. (C) JBP3-TAP mRNA levels for the T7-TR/JB3-MHTAP/ $\Delta j b p 3:: n e o$ clone grown in the presence or absence of Tet for the number of days indicated. mRNA levels are expressed as transcripts per million as determined by RNA-seq analysis using Geneious. (D) Box-and-whisker plots showing the median top strand coverage in the 5-kb region downstream of all 192 TTSs (All). Separate plots are shown for the 46 TTSs at cSSRs (Conv), 30 TTSs between head-to-tail PTUs (Uni), 39 TTSs immediately upstream of one or more RNA genes (RNA), 21 TTSs adjacent to a centromere (Cent), and 56 TTSs at telomeres (Telo). (E) Median top strand coverage at each nucleotide position in the $10 \mathrm{~kb}$ surrounding the 46 TTSs at cSSRs. The schematic represents the protein-coding genes associated with each strand at an "average" convergent TTS (cTTS). The second PTU at each cSSR is reoriented so that the genes are represented on the top strand. (F) Median top strand coverage at each nucleotide position in the $10 \mathrm{~kb}$ surrounding the 30 TTSs between unidirectional (head-to-tail) PTUs. The schematic represents the protein-coding genes associated with each strand at an "average" unidirectional TTS (UTTS). 


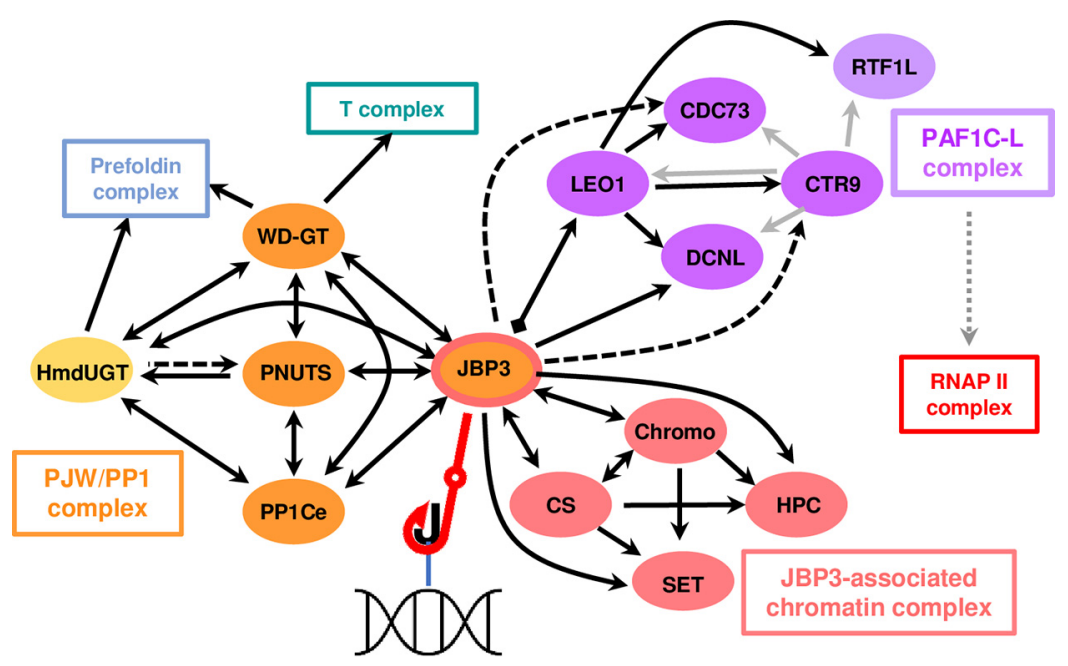

FIG 6 Network of interactions between JBP3-associated protein complexes. Solid lines denote proteins enriched in both replicates of the TAP-tag pulldowns, and dashed lines indicate proteins enriched in only one sample. Double-headed lines represent reciprocal enrichment with both proteins used as bait, while a diamond shape at one end of the line indicates that JBP3 was not detected in the LEO1 pulldown. Lines with a single arrowhead indicate that reciprocal enrichment was not attempted. Gray arrows represent interactions identified by copurification of proteins with CTR9 in T. brucei (38). Subunits within the three distinct JBP3-associated protein complexes are denoted by different colors, while the chaperone and RNA polymerase complexes are represented by boxes without individual components. The dotted line connecting the PAFC1-L complex to RNAP II reflects the interactions observed in other organisms. The interaction between JBP3 and base $J$ is marked by the red fishhook.

Tet $^{+}$and Tet $^{-}$samples (Fig. S4, left), except at cSSRs, where readthrough from the second PTU results in a substantial increase in antisense transcripts upstream of the TTS (Fig. S4B) due to readthrough from the convergent downstream PTU. A similar analysis of transcript abundance surrounding transcription start sites (TSSs) revealed no significant changes due to JBP3 depletion, except for a small increase in top (coding) strand coverage when PTUs were oriented unidirectionally (Fig. S5C and G), presumably due to readthrough from the preceding PTU. Importantly, there was little or no increase in bottom (noncoding) strand coverage upstream of most TSSs.

\section{DISCUSSION}

Using the trypanosomatid-specific GT, which carries out the second step of J biosynthesis, as an entrée to search for the molecular machinery associated with the regulation of transcription in Leishmania, we have identified a network of three protein complexes that contain conserved building blocks often used to assemble molecular machinery regulating transcription in other eukaryotes. A novel J-binding protein (JBP3) lies at the nexus of these complexes (Fig. 6) and provides new insight into the molecular mechanism(s) used to mediate transcription termination at the end of the polycistronic transcription units emblematic of these (and related trypanosomatid) parasites. We have shown that JBP3 plays a central role in controlling the termination of RNAP II transcription since the depletion of JBP3 leads to defects in transcriptional termination at the $3^{\prime}$ ends of PTUs in Leishmania (Fig. 5), just as it does in T. brucei (20). However, readthrough transcription is not seen to the same extent at all TTSs. The presence of RNAP III-transcribed RNA genes downstream of the TTS appears to effectively block RNAP II, as we have seen previously for jbp2 null mutants (11), and there is little or no readthrough at TTSs immediately upstream of centromeres and telomeres. This suggests that factors other than JBP3 may also play a role in reducing transcriptional readthrough at these loci. Alternatively, it is possible that the higher $\mathrm{J}$ content at centromeres and telomeres may more effectively "capture" what little JBP3 remains in 
the Tet $^{-}$cells. In contrast to the recent results from $T$. brucei (20), we find little evidence for antisense transcription at the $5^{\prime}$ ends of PTUs in Leishmania, even after the depletion of JBP3 levels. Divergent strand-switch regions (dSSRs), where adjacent PTUs are on opposite strands, tend to be smaller in Leishmania than those in T. brucei and lack the J-containing DNA found in the latter, suggesting that there may be little inappropriate antisense transcription in these regions. However, we find evidence that the depletion of JBP3 in Leishmania results in the upregulation of mRNA levels for proteincoding genes at the $3^{\prime}$ ends of PTUs (see Table S2 in the supplemental material). It is possible that this phenomenon is due to a more efficient polyadenylation of transcripts caused by the uncovering of cryptic trans-splicing sites downstream of the normal TTS. The toxic effects of a gradual accumulation of proteins from these mRNAs may be one explanation for the lag between the appearance of defects in transcription termination (day 2) and the decrease in the growth rate (day 4). This lag period also indicates that readthrough transcription is not an artifact of a reduced growth rate.

Our initial TAP-tagging experiments showed that GT associates (directly or indirectly) with four other proteins that resemble components of the PTW/PP1 complex, which plays a role in regulating transcriptional activity in other eukaryotes. The mammalian PTW/PP1 complex (containing PNUTS, TOX4, WDR82, and PP1C) has been implicated in numerous different cellular processes, including control of chromatin structure during cell cycle progression (27), repair of DNA damage by nonhomologous end joining (41), maintenance of telomere length (41), and developmental regulation of transcription (42). The Leishmania PJW/PP1 complex shows obvious parallels to the metazoan PTW/ PP1 complex by incorporating analogous (although not necessarily homologous) proteins, with JBP3 replacing the DNA-binding function of TOX4. However, there are some interesting differences between Leishmania PJW/PP1 and the homologous T. brucei complex, most notably the absence of PP1C in the latter, where the complex was therefore called PJW (20). This absence is intriguing in the light of a recent publication that showed that the mammalian PTW/PP1 complex dephosphorylates the transcription elongation factor Spt5, thereby causing the RNAP II transcription complex to decelerate within the termination zone downstream of poly(A) sites and allowing the Xrn2 exonuclease to "track down and dislodge" the polymerase from the DNA template (30). This suggests that Leishmania and American trypanosomes may use PJW/PP1 to dephosphorylate Spt5 and mediate transcription termination but that African trypanosomes do not use this mechanism. In addition, although T. brucei encodes an orthologue of GT, it was not found to be associated with the PJW complex, pointing to another potential biological difference from Leishmania (or perhaps merely reflecting our use of a more rapid and sensitive purification protocol). In mammalian PTW/PP1C, PNUTS contains not only the phosphatase inhibitor motif but also a nuclear localization signal and provides a "scaffold" for recruiting the other proteins. However, Leishmania PNUTS is much smaller and lacks an obvious nuclear localization signal, so it is possible that other proteins in the complex provide these functions. For example, JBP3 contains a domain with structural similarity to the $\mathrm{N}$-terminal TFIIS protein interaction domain found in mammalian PNUTS, and WD proteins can act as a scaffold in other complexes $(43,44)$.

JBP3 is also present in a complex (J3C) that contains proteins with domains suggesting a role in chromatin modification and/or remodeling. One protein (SET-J3C) contains a SET domain, which is often present in histone methyltransferases (HMTs) that modify lysine and arginine residues in histone proteins. HMTs usually also contain cysteine-rich pre-SET and post-SET domains that play a crucial role in substrate recognition and enzymatic activity by coordinating zinc ions. SET-J3C contains a Zn finger domain downstream of the SET domain (Fig. S3D) that may fulfill similar functions, suggesting that it is likely to have HMT activity. Two other proteins (ChromoJ3C and CS-J3C) contain Chromo and/or Chromo shadow domains typically involved in the recognition of the methylated lysine residues on histone tails and may be functional homologues of the metazoan heterochromatin protein 1 (HP1) and/or fission yeast Swi6, which contain similar domains and are involved in the repression of gene 
expression by heterochromatin (34). Thus, it is possible that J3C facilitates the repacking of chromatin at J-containing regions of the genome, rendering them heterochromatin-like and inaccessible to RNA polymerases. The function of the HPC-J3C subunit is unknown at this time, but the $T$. brucei orthologue (encoded by Tb927.1.4250) shows structural similarity to subunits from different protein complexes implicated in several processes, including histone modification and/or chromatin remodeling, and it localizes to the nucleus (45). This suggests that the primary function of HPC-J3C may be to serve as a scaffold for the recruitment and/or stabilization of the $\mathrm{J} 3 \mathrm{C}$ complex.

JBP3 also associates (albeit transiently and/or indirectly) with a third protein complex (PAF1C-L), providing another potential connection between $\mathrm{J}$ and the regulation of transcription. PAF1C-L contains proteins with functional domains similar to those in yeast and mammalian PAF1C, which associates with the large subunit of RNAP II (35, 46) and plays a critical role in transcription elongation and termination $(47,48)$. Four proteins (PAF1, CDC73, LEO1, and CTR9) are consistently found as components of PAF1C in all other eukaryotes, while RTF1 (49) and the WD40-containing protein Ski8/ WDR61 (50) show a less ubiquitous association. The parallels between the mammalian and trypanosomatid complexes are obvious since homologues of the CDC73, LEO1, and CTR9 subunits copurify with JBP3, and experiments performed by others in T. brucei showed that CTR9 has a tight association with LEO and CDC73 (38). Our TAP tag experiments using LEO1 as the bait protein showed enrichment of CDC73 and CTR9, along with a protein containing a Plus-3 domain similar to that in RTF1. Interestingly, the Plus-3 domain of RTF1 has been implicated in binding to Spt5 (51), which is tantalizing in light of the potential role for the PJW/PP1 complex in dephosphorylating this transcription factor (30). We (and others) failed to identify a convincing homologue of Ski8/WDR61 (or any other WD40 protein) in the PAF1C-L complex, but this protein is not tightly associated with mammalian PAF1C either. Remarkably, the namesake of the complex (PAF1) is absent from pulldowns of LEO and CTR9 in L. tarentolae and T. brucei, respectively, even though it (along with CTR9) is essential for the assembly of the complex in both yeast and humans (52). Moreover, extensive bioinformatic analysis of the trypanosomatid genomes failed to identify a homologue of PAF1, suggesting that it is truly absent from the Leishmania and Trypanosoma PAF1C-L complex. However, PAF1C-L contains an additional, trypanosomatid-specific component (DCNL) that has a putative protein-binding domain with structural similarity to the PONY/DCUN1 domain found in the eukaryotic DCN protein family. In other eukaryotes, DCN1 is required for the neddylation of cullin in SCF-type E3 ubiquitin ligase complexes that mark cellular proteins for proteasomal degradation (53). It is interesting to speculate that DCNL may be involved in PAF1C-L recruitment/function by interaction with N-terminal acetylated residues on histones and/or other chromatin-associated proteins. Whether DCNL functionally replaces PAF1 will remain an open question until its molecular function is dissected in more detail.

We have previously postulated that J might terminate transcription by directly preventing the progression of polymerase (11). The data presented here suggest three alternative, but not mutually exclusive, hypotheses based on the ability of JBP3 to bind base J. First, JBP3 may recruit the PJW/PP1 complex to J-containing regions, where it helps stall the elongating RNAP II by dephosphorylating Spt5 and causing the transcription complex to decelerate within the termination zone. Second, the J3C complex may enhance termination by modifying and/or remodeling the chromatin at J-containing regions, thereby preventing the passage of RNAP II. Third, we speculate that the interaction between JBP3 and PAF1C-L may also tether the RNAP II to the termination zone, where the DCNL subunit ubiquitinates the polymerase complex, promoting its degradation by the proteasome $(54,55)$. While disruption of the PJW complex in T. brucei (20) provides additional evidence for the first hypothesis, it remains to be seen whether disruption of J3C and PAF1C-L complexes also causes a defect in transcription termination. 
While our findings provide several novel insights into the role of base $J$ in transcription termination, they also raise several interesting questions. For example, why is GT part of the PJW/PP1 complex? One could envisage that the recruitment of the PJW/ PP1 complex to regions of the genome containing J recruits may allow the more efficient glucosylation of nearby $\mathrm{HmdU}$ residues. Our proteomics data suggest that JBP3 interacts to various degrees with three different protein complexes (J3C, PJW/PP1, and PAF1C-L). It will be important to understand how these interactions are regulated. Are the complexes present at the same region simultaneously, or are they temporally and/ or spatially segregated? For example, PJW/PP1 may be present at the ends of all PTUs, while J3C may be associated with only centromeres and/or telomeres. What histone modification/remodeling is mediated by $\mathrm{J} 3 \mathrm{C}$, and what role does this play in transcription termination? The availability of modern genome-wide approaches will no doubt provide the appropriate tools to answer these questions.

\section{MATERIALS AND METHODS}

Plasmid construction. To create an expression vector that expresses epitope-tagged transgenes in Leishmania, the MHTAP tag (which bears a Myc epitope, six histidines, a protein A domain, and calmodulin-binding peptide [CBP]) was amplified from the plasmid pLEW-MHTAP (56) with the primers MHTAPBamHI-S and MHTAP-Notl-AS (all primers used in this study are described in Table S3 in the supplemental material). Following cleavage with BamHI and Notl, the PCR fragment was inserted into Bglll- and Notl-digested pLEXSY-I-bleCherry3 (Jena Biosciences). The resulting plasmid (pLEXSY-MHTAP; Fig. S6) allows the TAP tagging of the introduced coding regions under the control of a Tet-regulated T7 promoter and insertion into the $O D C$ locus on chromosome 12 of $L$. tarentolae. We used a combination of previously reported data sets to identify the $5^{\prime}$ ends of $L$. tarentolae mRNAs as marked by the 39-bp splice leader sequence (11) and ribosome profiling data from Leishmania donovani (B. Jensen, R. Koren, G. Ramasamy, A. Haydock, A. Sekar, J. McDonald, D. Zilberstein, and P. J. Myler, unpublished data) to identify the correct coding DNA sequence (CDS) for bait proteins. CDSs were PCR amplified and digested with the restriction enzymes indicated in Table S3 prior to cloning.

Parasite strains and tissue culture. The Leishmania tarentolae Parrot-Tarll wild-type (WT) and T7-TR strains (Jena Bioscience) were grown in SDM-79 medium supplemented with 10\% fetal bovine serum. Strain T7-TR has constitutively expressed T7 RNA polymerase and Tet repressor (TetR) genes integrated into the ribosomal DNA (rDNA) locus, allowing for Tet-induced expression of integrated (or ectopically expressed) genes. Nourseothricin and hygromycin B were added to the medium at $100 \mu \mathrm{M}$ to maintain the expression of T7 RNA polymerase and TetR.

Tandem affinity purification of tagged protein complexes. Ten micrograms of the Swal-digested pLEXSY-MHTAP plasmid encoding a TAP-tagged protein was electroporated into the L. tarentolae WT and T7-TR cell lines as described previously (57), and transfectants were selected with $100 \mu \mathrm{g} / \mathrm{ml}$ bleomycin and maintained in $20 \mu \mathrm{g} / \mathrm{ml}$ bleomycin (plus nourseothricin and hygromycin B as described above). Proteins associated with the TAP-tagged "bait" were purified from $500 \mathrm{ml}$ of cells following culture overnight (in the presence of $2 \mu \mathrm{g} / \mathrm{ml}$ tetracycline for T7-TR transfectants) as described previously (56), except that NP-40 was omitted from the final four washes of the proteins on the calmodulin beads and from the calmodulin elution buffer. The protocol went from lysis of cells to purified samples within $6 \mathrm{~h}$. A sample from each pulldown (5\% of the total eluate) was separated by 4 to $20 \%$ SDS-PAGE, and proteins were visualized using SilverQuest stain (Thermo Fisher Scientific, Life Technologies). Fractions containing a protein with the predicted molecular weight of the bait (usually fractions 2 and 3) were pooled. The same fractions were pooled from mock TAPs of the control parental line not expressing any bait protein.

Western blotting. Proteins from transfected cells were separated by SDS-PAGE on 4-to-20\% gradient gels, transferred onto nitrocellulose, and detected with either mouse anti- $6 \times \mathrm{His}$ (Clontech) at $0.25 \mu \mathrm{g} / \mathrm{ml}$ or rabbit antibody against calmodulin-binding peptide (GenScript) at $0.1 \mu \mathrm{g} / \mathrm{ml}$, with rabbit antibody against $T$. brucei phosphoglycerate kinase serving as a control (58). Primary antibodies were detected with goat anti-rabbit lg conjugated with Alexa Fluor $680(50 \mathrm{ng} / \mathrm{ml})$ or goat anti-mouse lg conjugated with IRDye $800(25 \mathrm{ng} / \mathrm{ml})$ and imaged on the Li-Cor Odyssey CLX system.

Proteomic analysis. Pooled protein fractions were denatured with $6 \mathrm{M}$ urea, reduced with $5 \mathrm{mM}$ dithiothreitol, alkylated with $25 \mathrm{mM}$ iodoacetamide, and digested at $37^{\circ} \mathrm{C}$ for $3 \mathrm{~h}$ using 1:200 (wt/wt) endoproteinase Lys-C (Thermo Fisher Scientific). The urea was then diluted to $1.5 \mathrm{M}$, and samples were further digested at $37^{\circ} \mathrm{C}$ overnight with 1:25 (wt/wt) trypsin (Thermo Fisher Scientific). Proteinase activity was stopped with formic acid, and peptides were purified using $C_{18}$ reversed-phase chromatography (Waters), followed by hydrophilic interaction chromatography (HILIC; Nest Group). Purified peptides were separated by online nanoscale high-performance liquid chromatography (HPLC) (Easy-nLC II; Proxeon) with a $C_{18}$ reversed-phase column (Magic $C_{18} A Q 5 \mu \mathrm{m}, 100 \AA$ ) over an increasing 90-min gradient of 5 to $35 \%$ buffer B (100\% acetonitrile, $0.1 \%$ formic acid) at a flow rate of $300 \mathrm{nl} / \mathrm{min}$. Eluted peptides were analyzed with an Orbitrap Elite mass spectrometer (Thermo Fisher Scientific) operated in data-dependent mode, with the 15 most intense ions per MS1 survey scan selected for MS2 fragmentation by rapid collision-induced dissociation ( $\mathrm{rCID}$ ) (59). MS1 survey scans were performed in the Orbitrap instrument at a resolution of 240,000 at $\mathrm{m} / \mathrm{z} 400$ with charge state rejection enabled, while rCID MS2 
was performed in the dual linear ion trap with a minimum signal of 1,000. Dynamic exclusion was set to $15 \mathrm{~s}$.

Raw output data files were analyzed using MaxQuant (v1.5.3.30) (60) to search against a proteome predicted after resequencing and annotation of the L. tarentolae Parrot (LtaP) genome (A. Sur, J. McDonald, G. Ramasamy, and P. J. Myler, unpublished data). A reverse sequence decoy database was used to impose a strict $1 \%$ false discovery rate (FDR) cutoff. Label-free quantification was performed using the MaxLFQ algorithm (61), and further data processing was performed in Perseus (v1.5.3.1) (62) and Microsoft Excel. To avoid zero-value denominators, null values in the remaining data were replaced by imputation using the background signal within one experiment using Perseus. Nonparasite contaminants, decoys, and single-peptide identifications among all samples in an experiment were removed. Proteins were deemed to be part of a complex associated with the bait protein if at least two peptides were detected and the protein showed $>32$-fold $\left(\log _{2}\right.$ fold change of $>5$ ) enrichment (compared to the control not expressing the bait) in both replicates. In a few cases, proteins showing $>100$-fold enrichment in a single replicate only were also considered potential subunits of the complex. Proteins with 1,024 -fold $\left(\log _{2}\right.$ fold change of $<10$ ) less enrichment than the bait protein were assumed to be copurifying contaminants and (usually) ignored.

Bioinformatic analysis of protein function. Structure-based similarity searches for known domains were performed with HHpred (23). Domain boundaries for the JBP3 DNA-binding domain (DBD) were refined by aligning trypanosomatid sequences that clustered in the same OrthoMCL group as JBP3 with T-Coffee (63). Homology models of the JBP3 DBD domain using RosettaCM (64) were built with the $L$. tarentolae JBP1 structure (PDB accession number 2XSE) as a template. The top-scoring model covered residues 111 to 312 of the JBP3 DBD with a confidence score of 0.67 .

Deletion of JBP3 using SaCas9. JBP3 was deleted using Staphylococcus aureus Cas9 (SaCas9)directed cleavage of sites flanking the endogenous locus as described previously (40). Briefly, guide RNAs (gRNAs) directed at sites for SaCas9 cleavage were generated in vitro using T7 Megashort from Thermo Fisher from PCR-generated templates. The $5^{\prime}$ and $3^{\prime}$ gRNA sequences used were GATG TGAAACGCTAAGCAGTCCCGAGT and AGGAACGAAAGCACACAGCAGAGGAGT, where the protospaceradjacent motif (PAM) sites are underlined. Repair fragments containing a drug resistance gene were generated as described previously (65) from pTNeo or pTPuro templates using primers LtJBP3-up and LtJBP3-down. Heat-denatured guide RNA was complexed with $20 \mu \mathrm{g}$ SaCas9 recombinant protein at an equimolar ratio and incubated for $15 \mathrm{~min}$ at room temperature before mixing with $2 \mu \mathrm{g}$ of each repair fragment that had been ethanol precipitated and resuspend in Tb-BSF ( $T$. brucei bloodstream form) buffer (66). L. tarentolae T7-TR cells were grown overnight (in the presence of $2 \mu \mathrm{g} / \mathrm{ml}$ tetracycline for the latter), pelleted, washed with phosphate-buffered saline (PBS), and resuspended in $100 \mu \mathrm{l} \mathrm{Tb-BSF}$ buffer. For each transfection, $10^{6}$ cells were mixed with the SaCas9/guide RNA complexes and repair fragments and electroporated in an Amaxa Nucleofector using program X-001. Immediately following transfection, cells were split into three flasks. After allowing the cells to recover overnight, antibiotics were added to the flasks, one of which was grown in $10 \mu \mathrm{g} / \mathrm{ml} \mathrm{G} 418$, one of which was grown in $4 \mu \mathrm{g} /$ $\mathrm{ml}$ puromycin, and one of which was grown with both drugs. Deletion of the endogenous JBP3 gene(s) was confirmed by PCR amplification of genomic DNA using primers JBP3-M84P and JBP3-P2147M that flank the region being deleted. Clone cell lines were obtained by limiting dilution of the transfectants, and clones were retested for JBP3 deletion by PCR. While we were able to obtain clones where both endogenous copies of JBP3 were deleted by selection with either puromycin or neomycin, we were unable to obtain lines where both drugs were used.

RNA-seq analysis. RNA was isolated using TRIzol (Thermo Fisher Scientific) and resuspended in $10 \mathrm{mM}$ Tris (pH 7), and RNA quality was assessed using the Bioanalyzer 6000 Pico chip (Agilent). mRNA was isolated from $1 \mu \mathrm{g}$ total RNA using the New England BioLabs (NEB) poly(A) mRNA magnetic isolation module and prepared using the stranded RNA-seq protocol (67), modified for Leishmania as described previously (68). Libraries were sequenced on an Illumina HiSeq instrument, obtaining paired-end 150-bp reads. Reads were aligned against our in-house L. tarentolae genome with Bowtie2 (69) using the "very high sensitivity" parameter or the Geneious assembler (Geneious Prime 11.05) using the "low sensitivity/ fastest" option. Differential expression analysis was performed on the Geneious assemblies using the DESeq2 module to compare Tet ${ }^{+}$and Tet $^{-}$samples from days $2,3,4,6$, and 8 for replicate 1 and from days $2,3,4,5,7$, and 9 for replicate 2 . Strand-specific read coverage was calculated directly from BAM files of the Bowtie2 alignments using customized pysam scripts (https://github.com/pysam-developers/ pysam).

Data availability. Mass spectrometry data were deposited in the MassIVE database (https://massive .ucsd.edu/ProteoSAFe/static/massive.jsp) and can be accessed from ProteomeXchange under accession number PXD020779. RNA-seq data were deposited in the Sequence Read Archive (SRA) at the NCBI and can be accessed under BioProject accession number PRJNA657890.

\section{SUPPLEMENTAL MATERIAL}

Supplemental material is available online only.

FIG S1, PDF file, $0.02 \mathrm{MB}$.

FIG S2, PDF file, $0.1 \mathrm{MB}$.

FIG S3, PDF file, $0.4 \mathrm{MB}$.

FIG S4, PDF file, $0.3 \mathrm{MB}$.

FIG S5, PDF file, $0.3 \mathrm{MB}$. 
FIG S6, PDF file, $0.1 \mathrm{MB}$.

TABLE S1, PDF file, $0.1 \mathrm{MB}$.

TABLE S2, PDF file, $0.1 \mathrm{MB}$.

TABLE S3, PDF file, $0.1 \mathrm{MB}$.

DATA SET S1, XLSX file, 1.2 MB.

\section{ACKNOWLEDGMENTS}

We thank Lisa Jones of the Fred Hutchison Cancer Research Center Proteomics

Resources facility for assistance with performing LC-MS/MS analysis.

This work was supported in part by PHS grant R01 Al103858 (to P.J.M.) and contract

HHSN272201700059C (to P.J.M.) from the National Institute of Allergy and Infectious

Diseases.

Concept and experimental design were done by B.C.J. and P.J.M. Experiments were performed by B.C.J. and J.R.M. Proteomic analysis was done by M.A.G. and J.A.R. Protein modeling and sequence analysis were done by I.Q.P. Analysis of RNA-seq data were done by A.S. and P.J.M. The manuscript was written by B.C.J., M.P., and P.J.M. All authors have reviewed the manuscript.

We declare no competing interest.

\section{REFERENCES}

1. Thomas S, Green A, Sturm NR, Campbell DA, Myler PJ. 2009. Histone acetylations mark origins of polycistronic transcription in Leishmania major. BMC Genomics 10:152. https://doi.org/10.1186/1471-2164-10-152.

2. Martínez-Calvillo S, Yan S, Nguyen D, Fox M, Stuart K, Myler PJ. 2003. Transcription of Leishmania major Friedlin chromosome 1 initiates in both directions within a single region. Mol Cell 11:1291-1299. https://doi.org/ 10.1016/S1097-2765(03)00143-6.

3. Martinez-Calvillo S, Nguyen D, Stuart K, Myler PJ. 2004. Transcription initiation and termination on Leishmania major chromosome 3. Eukaryot Cell 3:506-517. https://doi.org/10.1128/ec.3.2.506-517.2004.

4. Gommers-Ampt JH, Van Leeuwen F, de Beer AL, Vliegenthart JF, Dizdaroglu M, Kowalak JA, Crain PF, Borst P. 1993. $\beta$-D-Glucosyl-hydroxymethyluracil: a novel modified base present in the DNA of the parasitic protozoan T. brucei. Cell 75:1129-1136. https://doi.org/10.1016/0092 $-8674(93) 90322-\mathrm{H}$

5. van Leeuwen F, Kieft R, Cross M, Borst P. 1998. Biosynthesis and function of the modified DNA base $\beta$-D-glucosyl-hydroxymethyluracil in Trypanosoma brucei. Mol Cell Biol 18:5643-5651. https://doi.org/10.1128/mcb.18 .10 .5643 .

6. van Leeuwen F, Taylor MC, Mondragon A, Moreau H, Gibson W, Kieft R, Borst P. 1998. $\beta$-D-Glucosyl-hydroxymethyluracil is a conserved DNA modification in kinetoplastid protozoans and is abundant in their telomeres. Proc Natl Acad Sci U S A 95:2366-2371. https://doi.org/10.1073/ pnas.95.5.2366.

7. Van Leeuwen F, Wijsman ER, Kuyl-Yeheskiely E, van der Marel GA, Van Boom JH, Borst P. 1996. The telomeric GGGTTA repeats of Trypanosoma brucei contain the hypermodified base $\mathrm{J}$ in both strands. Nucleic Acids Res 24:2476-2482. https://doi.org/10.1093/nar/24.13.2476.

8. van Leeuwen F, Wijsman ER, Kieft R, van der Marel GA, van Boom JH, Borst P. 1997. Localization of the modified base $J$ in telomeric VSG gene expression sites of Trypanosoma brucei. Genes Dev 11:3232-3241. https:// doi.org/10.1101/gad.11.23.3232.

9. van Leeuwen F, Kieft R, Cross M, Borst P. 2000. Tandemly repeated DNA is a target for the partial replacement of thymine by $\beta$-D-glucosyl-hydroxymethyluracil in Trypanosoma brucei. Mol Biochem Parasitol 109:133-145. https://doi.org/10.1016/s0166-6851(00)00247-4.

10. Cliffe LJ, Siegel TN, Marshall M, Cross GA, Sabatini R. 2010. Two thymidine hydroxylases differentially regulate the formation of glucosylated DNA at regions flanking polymerase II polycistronic transcription units throughout the genome of Trypanosoma brucei. Nucleic Acids Res 38:3923-3935. https://doi.org/10.1093/nar/gkq146.

11. van Luenen HG, Farris $C$, Jan $S$, Genest PA, Tripathi $P$, Velds A, Kerkhoven RM, Nieuwland M, Haydock A, Ramasamy G, Vainio S, Heidebrecht $T$, Perrakis A, Pagie L, van Steensel B, Myler PJ, Borst P. 2012. Glucosylated hydroxymethyluracil, DNA base $J$, prevents transcriptional readthrough in Leishmania. Cell 150:909-921. https://doi.org/10.1016/j.cell.2012.07 .030 .

12. Marques CA, Dickens NJ, Paape D, Campbell SJ, McCulloch R. 2015. Genome-wide mapping reveals single-origin chromosome replication in Leishmania, a eukaryotic microbe. Genome Biol 16:230. https://doi.org/10 .1186/s13059-015-0788-9.

13. Garcia-Silva MR, Sollelis L, MacPherson CR, Stanojcic S, Kuk N, Crobu L, Bringaud F, Bastien P, Pages M, Scherf A, Sterkers Y. 2017. Identification of the centromeres of Leishmania major: revealing the hidden pieces. EMBO Rep 18:1968-1977. https://doi.org/10.15252/embr.201744216.

14. Sekar A, Merritt C, Baugh L, Stuart K, Myler PJ. 2014. Tb927.10.6900 encodes the glucosyltransferase involved in synthesis of base $\mathrm{J}$ in Trypanosoma brucei. Mol Biochem Parasitol 196:9-11. https://doi.org/10.1016/j .molbiopara.2014.07.005.

15. Heidebrecht T, Christodoulou E, Chalmers MJ, Jan S, Ter Riet B, Grover RK, Joosten RP, Littler D, van Luenen $H$, Griffin PR, Wentworth $P$, Jr, Borst $P$, Perrakis A. 2011. The structural basis for recognition of base $J$ containing DNA by a novel DNA binding domain in JBP1. Nucleic Acids Res 39:5715-5728. https://doi.org/10.1093/nar/gkr125.

16. DiPaolo C, Kieft R, Cross M, Sabatini R. 2005. Regulation of trypanosome DNA glycosylation by a SWI2/SNF2-like protein. Mol Cell 17:441-451. https://doi.org/10.1016/j.molcel.2004.12.022.

17. Cliffe LJ, Kieft R, Southern T, Birkeland SR, Marshall M, Sweeney K, Sabatini R. 2009. JBP1 and JBP2 are two distinct thymidine hydroxylases involved in $\mathrm{J}$ biosynthesis in genomic DNA of African trypanosomes. Nucleic Acids Res 37:1452-1462. https://doi.org/10.1093/nar/gkn1067.

18. Genest PA, ter Riet B, Dumas C, Papadopoulou B, van Luenen HG, Borst P. 2005. Formation of linear inverted repeat amplicons following targeting of an essential gene in Leishmania. Nucleic Acids Res 33:1699-1709. https:// doi.org/10.1093/nar/gki304.

19. Genest PA, Baugh L, Taipale A, Zhao W, Jan S, van Luenen HG, Korlach J, Clark T, Luong K, Boitano M, Turner S, Myler PJ, Borst P. 2015. Defining the sequence requirements for the positioning of base $J$ in DNA using SMRT sequencing. Nucleic Acids Res 43:2102-2115. https://doi.org/10.1093/ nar/gkv095.

20. Kieft R, Zhang $Y$, Marand AP, Moran JD, Bridger R, Wells L, Schmitz RJ, Sabatini R. 2020. Identification of a novel base J binding protein complex involved in RNA polymerase II transcription termination in trypanosomes. PLoS Genet 16:e1008390. https://doi.org/10.1371/journal.pgen.1008390.

21. Bollen M, Peti W, Ragusa MJ, Beullens M. 2010. The extended PP1 toolkit: designed to create specificity. Trends Biochem Sci 35:450-458. https://doi .org/10.1016/j.tibs.2010.03.002.

22. Lesage B, Beullens M, Pedelini L, Garcia-Gimeno MA, Waelkens E, Sanz P, Bollen M. 2007. A complex of catalytically inactive protein phosphatase-1 sandwiched between Sds22 and inhibitor-3. Biochemistry 46:8909-8919. https://doi.org/10.1021/bi7003119. 
23. Zimmermann L, Stephens A, Nam SZ, Rau D, Kubler J, Lozajic M, Gabler F, Soding J, Lupas AN, Alva V. 2018. A completely reimplemented MPI bioinformatics toolkit with a new HHpred server at its core. J Mol Biol 430:2237-2243. https://doi.org/10.1016/j.jmb.2017.12.007.

24. Kim YM, Watanabe T, Allen PB, Kim YM, Lee SJ, Greengard P, Nairn AC, Kwon YG. 2003. PNUTS, a protein phosphatase 1 (PP1) nuclear targeting subunit. Characterization of its PP1- and RNA-binding domains and regulation by phosphorylation. J Biol Chem 278:13819-13828. https://doi.org/ 10.1074/jbc.M209621200.

25. Kreivi JP, Trinkle-Mulcahy L, Lyon CE, Morrice NA, Cohen P, Lamond AI. 1997. Purification and characterisation of p99, a nuclear modulator of protein phosphatase 1 activity. FEBS Lett 420:57-62. https://doi.org/10 .1016/s0014-5793(97)01485-3.

26. Allen PB, Kwon YG, Nairn AC, Greengard P. 1998. Isolation and characterization of PNUTS, a putative protein phosphatase 1 nuclear targeting subunit. J Biol Chem 273:4089-4095. https://doi.org/10.1074/jbc.273.7.4089.

27. Lee JH, You J, Dobrota E, Skalnik DG. 2010. Identification and characterization of a novel human PP1 phosphatase complex. J Biol Chem 285:2446624476. https://doi.org/10.1074/jbc.M110.109801.

28. Choy MS, Hieke M, Kumar GS, Lewis GR, Gonzalez-DeWhitt KR, Kessler RP, Stein BJ, Hessenberger M, Nairn AC, Peti W, Page R. 2014. Understanding the antagonism of retinoblastoma protein dephosphorylation by PNUTS provides insights into the PP1 regulatory code. Proc Natl Acad Sci U S A 111:4097-4102. https://doi.org/10.1073/pnas.1317395111.

29. Landsverk HB, Kirkhus M, Bollen M, Kuntziger T, Collas P. 2005. PNUTS enhances in vitro chromosome decondensation in a PP1-dependent manner. Biochem J 390:709-717. https://doi.org/10.1042/BJ20050678.

30. Cortazar MA, Sheridan RM, Erickson B, Fong N, Glover-Cutter K, Brannan K, Bentley DL. 2019. Control of RNA Pol II speed by PNUTS-PP1 and Spt5 dephosphorylation facilitates termination by a "sitting duck torpedo" mechanism. Mol Cell 76:896-908.e4. https://doi.org/10.1016/j.molcel .2019.09.031.

31. Dillon SC, Zhang X, Trievel RC, Cheng X. 2005. The SET-domain protein superfamily: protein lysine methyltransferases. Genome Biol 6:227. https://doi.org/10.1186/gb-2005-6-8-227.

32. Paro R. 1990. Imprinting a determined state into the chromatin of Drosophila. Trends Genet 6:416-421. https://doi.org/10.1016/0168-9525(90)90303-n.

33. Paro R, Hogness DS. 1991. The Polycomb protein shares a homologous domain with a heterochromatin-associated protein of Drosophila. Proc Natl Acad Sci U S A 88:263-267. https://doi.org/10.1073/pnas.88.1.263.

34. Aasland R, Stewart AF. 1995. The chromo shadow domain, a second chromo domain in heterochromatin-binding protein 1, HP1. Nucleic Acids Res 23:3168-3173. https://doi.org/10.1093/nar/23.16.3168.

35. Rozenblatt-Rosen O, Hughes CM, Nannepaga SJ, Shanmugam KS, Copeland TD, Guszczynski T, Resau JH, Meyerson M. 2005. The parafibromin tumor suppressor protein is part of a human Paf1 complex. Mol Cell Biol 25:612-620. https://doi.org/10.1128/MCB.25.2.612-620.2005.

36. Pavri R, Zhu B, Li G, Trojer P, Mandal S, Shilatifard A, Reinberg D. 2006. Histone $\mathrm{H} 2 \mathrm{~B}$ monoubiquitination functions cooperatively with $\mathrm{FACT}$ to regulate elongation by RNA polymerase II. Cell 125:703-717. https://doi.org/ 10.1016/j.cell.2006.04.029.

37. Krogan NJ, Kim M, Ahn SH, Zhong G, Kobor MS, Cagney G, Emili A, Shilatifard A, Buratowski S, Greenblatt JF. 2002. RNA polymerase II elongation factors of Saccharomyces cerevisiae: a targeted proteomics approach. Mol Cell Biol 22:6979-6992. https://doi.org/10.1128/mcb.22.20.6979-6992 .2002 .

38. Ouna BA, Nyambega B, Manful T, Helbig C, Males M, Fadda A, Clayton C. 2012. Depletion of trypanosome CTR9 leads to gene expression defects. PLoS One 7:e34256. https://doi.org/10.1371/journal.pone.0034256.

39. Scott DC, Hammill JT, Min J, Rhee DY, Connelly M, Sviderskiy VO, Bhasin D, Chen Y, Ong SS, Chai SC, Goktug AN, Huang G, Monda JK, Low J, Kim HS, Paulo JA, Cannon JR, Shelat AA, Chen T, Kelsall IR, Alpi AF, Pagala V, Wang X, Peng J, Singh B, Harper JW, Schulman BA, Guy RK. 2017. Blocking an N-terminal acetylation-dependent protein interaction inhibits an E3 ligase. Nat Chem Biol 13:850-857. https://doi.org/10.1038/nchembio .2386 .

40. Soares Medeiros LC, South L, Peng D, Bustamante JM, Wang W, Bunkofske M, Perumal N, Sanchez-Valdez F, Tarleton RL. 2017. Rapid, selection-free, high-efficiency genome editing in protozoan parasites using CRISPR-Cas9 ribonucleoproteins. mBio 8:e01788-17. https://doi.org/10.1128/mBio.01788 -17 .

41. Kim H, Lee OH, Xin H, Chen LY, Qin J, Chae HK, Lin SY, Safari A, Liu D, Songyang Z. 2009. TRF2 functions as a protein hub and regulates telomere maintenance by recognizing specific peptide motifs. Nat Struct Mol Biol 16:372-379. https://doi.org/10.1038/nsmb.1575.

42. Ciurciu A, Duncalf L, Jonchere V, Lansdale N, Vasieva O, Glenday P, Rudenko A, Vissi E, Cobbe N, Alphey L, Bennett D. 2013. PNUTS/PP1 regulates RNAPIImediated gene expression and is necessary for developmental growth. PLoS Genet 9:e1003885. https://doi.org/10.1371/journal.pgen.1003885.

43. Stirnimann CU, Petsalaki E, Russell RB, Muller CW. 2010. WD40 proteins propel cellular networks. Trends Biochem Sci 35:565-574. https://doi.org/ 10.1016/j.tibs.2010.04.003.

44. Schapira M, Tyers M, Torrent M, Arrowsmith CH. 2017. WD40 repeat domain proteins: a novel target class? Nat Rev Drug Discov 16:773-786. https://doi.org/10.1038/nrd.2017.179.

45. Dean S, Sunter JD, Wheeler RJ. 2017. TrypTag.org: a trypanosome genomewide protein localisation resource. Trends Parasitol 33:80-82. https://doi .org/10.1016/j.pt.2016.10.009.

46. Yart A, Gstaiger M, Wirbelauer C, Pecnik M, Anastasiou D, Hess D, Krek W. 2005. The HRPT2 tumor suppressor gene product parafibromin associates with human PAF1 and RNA polymerase II. Mol Cell Biol 25:5052-5060. https://doi.org/10.1128/MCB.25.12.5052-5060.2005.

47. Tomson BN, Arndt KM. 2013. The many roles of the conserved eukaryotic Paf1 complex in regulating transcription, histone modifications, and disease states. Biochim Biophys Acta 1829:116-126. https://doi.org/10.1016/ j.bbagrm.2012.08.011.

48. Van Oss SB, Cucinotta CE, Arndt KM. 2017. Emerging insights into the roles of the Paf1 complex in gene regulation. Trends Biochem Sci 42:788-798. https://doi.org/10.1016/j.tibs.2017.08.003.

49. Mueller CL, Jaehning JA. 2002. Ctr9, Rtf1, and Leo1 are components of the Paf1/RNA polymerase II complex. Mol Cell Biol 22:1971-1980. https://doi .org/10.1128/mcb.22.7.1971-1980.2002.

50. Zhu B, Mandal SS, Pham AD, Zheng Y, Erdjument-Bromage H, Batra SK, Tempst P, Reinberg D. 2005. The human PAF complex coordinates transcription with events downstream of RNA synthesis. Genes Dev 19:1668-1673. https://doi.org/10.1101/gad.1292105.

51. Mayekar MK, Gardner RG, Arndt KM. 2013. The recruitment of the Saccharomyces cerevisiae Paf1 complex to active genes requires a domain of Rtf1 that directly interacts with the Spt4-Spt5 complex. Mol Cell Biol 33:3259-3273. https://doi.org/10.1128/MCB.00270-13.

52. Xie Y, Zheng M, Chu X, Chen Y, Xu H, Wang J, Zhou H, Long J. 2018. Paf1 and Ctr9 subcomplex formation is essential for Paf1 complex assembly and functional regulation. Nat Commun 9:3795. https://doi.org/10.1038/ s41467-018-06237-7.

53. Kurz T, Ozlu N, Rudolf F, O'Rourke SM, Luke B, Hofmann K, Hyman AA, Bowerman B, Peter M. 2005. The conserved protein DCN-1/Dcn 1p is required for cullin neddylation in $C$. elegans and $S$. cerevisiae. Nature 435:1257-1261. https://doi.org/10.1038/nature03662.

54. Nakazawa Y, Hara Y, Oka Y, Komine O, van den Heuvel D, Guo C, Daigaku $Y$, Isono $M$, He $Y$, Shimada M, Kato K, Jia N, Hashimoto S, Kotani Y, Miyoshi Y, Tanaka M, Sobue A, Mitsutake N, Suganami T, Masuda A, Ohno K, Nakada S, Mashimo T, Yamanaka K, Luijsterburg MS, Ogi T. 2020. Ubiquitination of DNA damage-stalled RNAPII promotes transcription-coupled repair. Cell 180:1228-1244.e24. https://doi.org/10.1016/j.cell.2020.02.010.

55. Tufegdzic Vidakovic A, Mitter R, Kelly GP, Neumann M, Harreman M, Rodriguez-Martinez M, Herlihy A, Weems JC, Boeing S, Encheva V, Gaul L, Milligan L, Tollervey D, Conaway RC, Conaway JW, Snijders AP, Stewart A, Svejstrup JQ. 2020. Regulation of the RNAPII pool is integral to the DNA damage response. Cell 180:1245-1261.e21. https://doi.org/10.1016/j.cell .2020.02.009.

56. Jensen BC, Kifer CT, Brekken DL, Randall AC, Wang Q, Drees BL, Parsons M. 2007. Characterization of protein kinase CK2 from Trypanosoma brucei. Mol Biochem Parasitol 151:28-40. https://doi.org/10.1016/j.molbiopara .2006.10.002.

57. Kapler GM, Zhang K, Beverley SM. 1990. Nuclease mapping and DNA sequence analysis of transcripts from the dihydrofolate reductase-thymidylate synthase $(\mathrm{R})$ region of Leishmania major. Nucleic Acids Res 18:6399-6408. https://doi.org/10.1093/nar/18.21.6399.

58. Parker HL, Hill T, Alexander K, Murphy NB, Fish WR, Parsons M. 1995. Three genes and two isozymes: gene conversion and the compartmentalization and expression of the phosphoglycerate kinases of Trypanosoma (Nannomonas) congolense. Mol Biochem Parasitol 69:269-279. https://doi.org/10 .1016/0166-6851(94)00208-5.

59. Michalski A, Damoc E, Lange O, Denisov E, Nolting D, Muller M, Viner R, Schwartz J, Remes P, Belford M, Dunyach J-J, Cox J, Horning S, Mann M, Makarov A. 2012. Ultra high resolution linear ion trap Orbitrap mass spectrometer (Orbitrap Elite) facilitates top down LC MS/MS and versatile 
peptide fragmentation modes. Mol Cell Proteomics 11:0111.013698. https://doi.org/10.1074/mcp.0111.013698.

60. Cox J, Mann M. 2008. MaxQuant enables high peptide identification rates, individualized p.p.b.-range mass accuracies and proteome-wide protein quantification. Nat Biotechnol 26:1367-1372. https://doi.org/10.1038/nbt.1511.

61. Cox J, Hein MY, Luber CA, Paron I, Nagaraj N, Mann M. 2014. Accurate proteome-wide label-free quantification by delayed normalization and maximal peptide ratio extraction, termed MaxLFQ. Mol Cell Proteomics 13:2513-2526. https://doi.org/10.1074/mcp.M113.031591.

62. Tyanova S, Temu T, Sinitcyn P, Carlson A, Hein MY, Geiger T, Mann M, Cox J. 2016. The Perseus computational platform for comprehensive analysis of (prote)omics data. Nat Methods 13:731-740. https://doi.org/10.1038/ nmeth.3901.

63. Di Tommaso P, Moretti S, Xenarios I, Orobitg M, Montanyola A, Chang JM, Taly JF, Notredame C. 2011. T-Coffee: a Web server for the multiple sequence alignment of protein and RNA sequences using structural information and homology extension. Nucleic Acids Res 39:W13-W17. https://doi.org/10.1093/nar/gkr245.
64. Song Y, DiMaio F, Wang RY, Kim D, Miles C, Brunette T, Thompson J, Baker D. 2013. High-resolution comparative modeling with RosettaCM. Structure 21:1735-1742. https://doi.org/10.1016/j.str.2013.08.005.

65. Beneke T, Madden R, Makin L, Valli J, Sunter J, Gluenz E. 2017. A CRISPR Cas9 high-throughput genome editing toolkit for kinetoplastids. R Soc Open Sci 4:170095. https://doi.org/10.1098/rsos.170095.

66. Schumann Burkard G, Jutzi P, Roditi I. 2011. Genome-wide RNAi screens in bloodstream form trypanosomes identify drug transporters. Mol Biochem Parasitol 175:91-94. https://doi.org/10.1016/j.molbiopara.2010.09 .002 .

67. Hunt AG. 2015. A rapid, simple, and inexpensive method for the preparation of strand-specific RNA-Seq libraries. Methods Mol Biol 1255:195-207. https://doi.org/10.1007/978-1-4939-2175-1_17.

68. Myler PJ, McDonald JA, Alcolea PJ, Sur A. 2019. Quantitative RNA analysis using RNA-seq. Methods Mol Biol 1971:95-108. https://doi.org/10.1007/ 978-1-4939-9210-2_4.

69. Langmead B, Salzberg SL. 2012. Fast gapped-read alignment with Bowtie 2. Nat Methods 9:357-359. https://doi.org/10.1038/nmeth.1923. 